Dominique Andolfatto, professeur de science politique,

Université de Bourgogne Franche-Comté

Dominique.Andolfatto@u-bourgogne.fr

Dominique Labbé, chercheur associé au Pacte-CNRS,

Université de Grenoble-Alpes

dominique.labbe@umrpacte.fr

\title{
Un échec français : la démocratie sociale
}

Article paru dans Le Débat. 2019/4, 206, p. 84-92.

\section{Résumé}

En 2008, une loi réformant le droit syndical visait à instaurer une démocratie sociale en France. Dix ans après, le constat d'échec repose sur le recul continu de la participation aux élections professionnelles, le fossé grandissant entre représentants et représentés, la poursuite du recul de la syndicalisation. Dans le même temps, les appareils syndicaux n'ont cessé de grossir et sont de plus en plus dépendants du financement public et des aides des grandes entreprises. Cette situation a conduit à une crise de la représentation qui s'est manifestée lors des événements de l'hiver 2018-2019 et du printemps 2019.

Manuscrit des auteurs. Toute citation doit se faire à partir de la revue Le Débat (consultable sur CAIRN) 
Les événements spectaculaires qui ont secoué la France à partir de novembre 2018 ont révélé encore une fois la relation difficile qu'entretient une proportion importante de Français avec leurs dirigeants. Ce fut une nouvelle illustration d'un paradoxe hexagonal : peu de pays, et peut-être aucun, n'a autant de "représentants" et pourtant beaucoup de Français se sentent mal ou pas représentés. Du point de vue politique, au plan national, il y a trois assemblées, nombreuses et bien dotées (Assemblée nationale, Sénat, Conseil économique, social et environnemental) ${ }^{1}$, et on retrouve des assemblées - également nombreuses - au niveau régional, départemental et communal ${ }^{2}$. De même, au plan social, il y a en France un grand nombre d'organismes censés être dirigés par des représentants des usagers - telles les caisses de la sécurité sociale ou le système d'assurance-chômage. Mais surtout, on trouve de nombreux représentants des salariés, depuis les établissements jusqu'aux grands groupes mais aussi les collectivités territoriales, les hôpitaux, les ministères... Dans le secteur marchand et associatif (soit un peu plus de 19 millions d'emplois) environ 600000 salariés (plus de 3\% des salariés) disposent d'au moins un mandat représentatif : élus du personnel ou délégués syndicaux ${ }^{3}$. Dans l'ensemble du pays, plus de 40000 salariés et fonctionnaires en ont fait leur activité unique.

L'émergence de ce groupe social est passée inaperçue. Plus au fond, la France a évolué d'un modèle d'action collective reposant sur les adhérents et les militants à un autre dominé par des professionnels de la représentation dotés de solides ressources institutionnelles mais aux bases électorales fragiles.

En 2008, une loi a pris acte de cette évolution et a affirmé "rénover la démocratie sociale" en fondant la légitimité des syndicats sur leurs résultats électoraux. En dépit de propos publics lénifiants, cette réforme est loin d'avoir produit les effets attendus comme le montrent plusieurs symptômes parmi lesquels : une participation électorale problématique, un taux de syndicalisation en recul et des organisations qui se réduisent de plus en plus à des appareils. Ces caractéristiques pourraient expliquer pourquoi les syndicats ont paru hors du coup lors des événements de l'hiver 2018-2019.

\footnotetext{
${ }^{1}$ Soit, au total, 1158 représentants au plan national.

$2 \mathrm{Au}$ total, plus de 511000 élus locaux (y compris les membres des conseils économiques, sociaux et environnementaux régionaux).

${ }^{3}$ Les représentants du personnel dans l'entreprise : des salariés comme les autres ? DARES Analyses, Janvier 2019, n 002 (consultable en ligne).
} 


\section{Une participation électorale problématique}

Depuis une loi d'août 2008, la représentativité des syndicats, et donc leur capacité à négocier au nom des salariés, dépend de leur audience aux élections professionnelles dans les entreprises, les branches d'activité et au niveau national interprofessionnel. Des règles assez comparables ont été introduites dans la fonction publique en 2010. Or ces réformes qui devaient permettre aux organisations syndicales de renouer avec les salariés, en substituant des électeurs à des adhérents défaillants, n'ont pas atteint leurs objectifs auprès des 19 millions de salariés du secteur privé ni auprès des 5,5 millions de fonctionnaires ${ }^{4}$.

Pour le secteur privé, les élections professionnelles sur le lieu du travail sont au cœur du dispositif. Paradoxalement, ces résultats électoraux sont devenus plus confidentiels qu'antérieurement. Seuls des résultats nationaux sont publiés une fois tous les quatre ans (on parle officiellement de "mesure de l'audience pour la représentativité syndicale") ${ }^{5}$. On ne dispose plus de résultats par branche d'activité (bien que la représentativité soit aussi mesurée à ce niveau), par région ou par taille d'entreprise ${ }^{6}$. Pour les années 2013-2016, dernière période pour laquelle le ministère du Travail a publié cette "mesure", il y aurait eu 42,8\% de votants (pourcentage étonnamment identique, à une décimale près, à celui de la première mesure d'audience, pour la période 2009-2012). Bref, les salariés français auraient moins voté que les électeurs français lors des dernières élections législatives (48,7\%), niveau de participation qui constituait un record historique de faiblesse ${ }^{7}$. Si l'on tient compte des bulletins blancs et nuls, c'est même seulement 39,6\% qui a exprimé un choix (et seulement $34,4 \%$ en faveur des cinq confédérations historiques: CGT, CFDT, FO, CFTC, CGC). Ajoutons que, faute d'élections dans certaines entreprises, et notamment celles de petite taille, tous les salariés ne peuvent participer à cette "mesure de l'audience" syndicale. De fait, celleci n'a couvert que 13,2 millions de salariés sur les quelque 19 millions que compte le secteur privé. Rapporté à ce dernier chiffre, le soutien aux diverses organisations syndicales s'établit

\footnotetext{
${ }^{4}$ 5,7 millions en incluant les emplois aidés (selon le Rapport annuel sur l'état de la fonction publique, édition 2018).

${ }^{5}$ Communiqué du ministère du Travail (DGT), 31 mars 2017.

${ }^{6}$ Le ministère du Travail a mis en place un serveur (Mars) pour les résultats des élections professionnelles par entreprise. Depuis fin 2018, on trouve ces résultats bruts sur la plateforme ouverte des données publiques françaises. Pour une première analyse, voir: Tristan Haute, Evolution du paysage syndical et du salariat : analyse des élections aux comités d'entreprise (2009-2018), La Revue de l'IRES, n 94-95, 2018/1, p. 59.

7 Pour s'en tenir au premier tour des élections législatives puisque la "mesure de l'audience pour la représentativité syndicale" se limite au seul premier tour des élections professionnelles (et ignorant les seconds tours).
} 
donc à moins de trois salariés sur dix (24,0\% pour les "cinq" représentatives). Autrement dit, les trois-quarts du salariat échappent aux organisations qui polarisent l'actualité sociale et participent à l'élaboration des réformes sociales.

Outre les résultats des élections professionnelles dans les entreprises, la "mesure de l'audience" syndicale intègre les résultats des élections des TPE (très petites entreprises) et du collège salarié de la production agricole (l'un des collèges des élections aux chambres d'agriculture). Le détail des résultats de ces deux derniers scrutins est partiellement disponible $^{8}$. L'un et l'autre traduisent l'incapacité des syndicats à amener ces salariés aux urnes quand le scrutin ne se déroule pas sur le lieu du travail. En effet, lors des dernières élections des TPE (décembre 2016 et janvier 2017), la participation n'a mobilisé que 7,3\% des électeurs (10,4\% lors de la première consultation, en 2012). S'agissant des salariés de l'agriculture, les résultats ne sont guère plus élevés : 10,2\% en 2019 (16,1\% en 2013). Autrement dit, ces élections se caractérisent par une abstention massive et en forte progression. Elles montrent le caractère illusoire des prétentions visant à fonder la représentativité syndicale - et la démocratie sociale - sur des scrutins comparables aux consultations politiques. Logiquement, bien des salariés des petites entreprises ou du secteur agricole éprouvent le sentiment légitime d'être oubliés.

Enfin les trois fonctions publiques - Etat, collectivités locales, hôpitaux, soit 5,5 millions de salariés - ont longtemps été vues comme une sorte de sanctuaire pour le syndicalisme. Pourtant, lors du dernier scrutin, dans les trois fonctions publiques, la participation est passée globalement sous la barre des 50\% des électeurs inscrits (précisément 49,8\% contre 52,8\% lors de la consultation précédente, en 2014). C'est dans la fonction publique hospitalière que cette participation est la plus faible $(44,1 \%)$. A l'Assistance Publique - Hôpitaux de Paris (72 000 titulaires) elle est tombée à 29\% et à 27\% aux Hospices civils de Lyon.

Il y a un demi-siècle, $82 \%$ des fonctionnaires votaient à ces élections. Dans la fonction publique d'Etat, la participation était encore de 71,2\% en 2003-2005. En treize ans, l'abstention a donc progressé de $44 \%$.

Manifestement la réforme du dialogue social a produit l'inverse de ce qui était recherché : au lieu de refonder la légitimité des organisations syndicales, elle a creusé le fossé

\footnotetext{
${ }^{8}$ Le ministère du Travail publie les résultats des élections des TPE par département et au niveau national (avant de les intégrer à la "mesure de la représentativité"). Par contre, le ministère de l'Agriculture n'a pas rendu public le détail des résultats du collège salarié de la production agricole. Ce détail a été communiqué aux différentes organisations et nous remercions la CFDT de nous les avoir transmis.
} 
entre représentés et représentants. Non seulement les électeurs n'ont pas remplacé les adhérents, mais le nombre de ces derniers continue à baisser.

\section{Une sous-syndicalisation toujours plus importante}

En 2008, les promoteurs de la réforme de la représentativité syndicale affirmaient que, en plaçant l'élection au cœur des relations professionnelles, on allait rapprocher les syndicalistes des salariés, stopper la désyndicalisation, obliger les organisations à se regrouper, et favoriser de nouvelles adhésions. Dix ans après, aucun de ces objectifs n'est atteint. Avec l'effondrement de la participation électorale, l'échec le plus flagrant concerne la syndicalisation.

En 2016, d'après une enquête de l'INSEE, auprès d'un échantillon représentatif des salariés des entreprises de plus de 10 salariés, la France est lanterne rouge de tous les grands pays comparables ${ }^{9}$. Pourtant, le ministère du travail en tirait deux conclusions étonnantes. D'une part, il affirmait que la syndicalisation serait finalement plus élevée qu'on le pensait auparavant $^{10}(11 \%$ contre $8 \%)$ et que la désyndicalisation serait stoppée. D’autre part, toujours selon le ministère, la syndicalisation en France aurait toujours été faible, ce qui justifie les aides et les réformes engagées.

La manière dont les statisticiens officiels parviennent à ces conclusions est pour le moins étonnante.

Pour la situation présente, l'enquête utilisée en 2016 posait deux questions relatives à la syndicalisation. A la première question, 7\% des enquêtés se déclaraient adhérents à un syndicat et à la seconde $11 \%$. L'analyse globale du ministère du Travail retenait finalement ce dernier chiffre, permettant d'affirmer que la désyndicalisation était stoppée. Cependant, pour le détail de cette syndicalisation (par âge ou par secteur économique), c'était souvent la question à $7 \%$ qui semblait mobilisée. De plus, les auteurs de la note ont exclu les chômeurs du dénominateur du taux de syndicalisation au contraire de ce qui est pratiqué habituellement, ce qui revient à augmenter le taux de plus de $10 \%$.

Quoiqu'il en soit de ces incohérences et obscurités, les informations en provenance des organisations syndicales montrent que l'optimisme du ministère du Travail est mal fondé. Ainsi, en mai 2018, la CFDT a « corrigé » ses effectifs « officiels » à la baisse - de 860000 à

\footnotetext{
${ }^{9}$ La syndicalisation en France: Des salariés deux fois plus syndiqués dans la fonction publique. DARES Analyses, mai 2016, $\mathrm{n}^{\circ} 025$.

${ }^{10}$ Mythes et réalités de la syndicalisation en France, DARES, Premières synthèses, 2004, $\mathrm{n}^{\circ} 44.2$.
} 
623000 - tout en prétendant que l'essentiel de cette baisse $(30 \%)$ serait due à un « changement de méthodologie dans les décompte des adhérents ». De son côté la CGT a admis avoir perdu 30000 adhérents entre 2012 et 2016. Avant sa démission forcée du secrétariat général de FO, P. Pavajot avait laissé entendre que le chiffre de 500000 adhérents revendiqués officieusement par cette centrale était exagéré ${ }^{11}$. Enfin, aucune autre organisation syndicale n'a fait état d'une augmentation de ses effectifs. Comme, dans le même temps, la population active salariée continue à augmenter à un rythme assez rapide, il est impossible que le taux de syndicalisation puisse rester stable et encore moins se redresser. L'artefact statistique est donc certain et l'on peut se demander pourquoi la presse et la plupart des chercheurs ont repris ces informations sans un minimum de recul critique.

La seconde thèse du ministère du Travail - la syndicalisation a toujours été faible en France - ne tient pas davantage. En effet, entre 1987 et 2006, ce ministère a commandité une série d'études ${ }^{12}$ qui - selon les propres termes d'un statisticien de ce ministère ${ }^{13}$ - : «ont permis d'établir les séries de référence » concernant l'évolution des effectifs syndiqués de la Libération au début des années 2000. Or en 2004 puis en 2016, les statisticiens du ministère du Travail ont décidé d'amputer la série 1947-1996 de près d'un tiers ${ }^{14}$ en même temps qu'ils remontaient les taux les plus récents. Ces "enjolivements" ont été reçus avec satisfaction à la fois par ceux qui, dans un pays à la tradition révolutionnaire comme la France, attendent avec

\footnotetext{
${ }^{11}$ Cécile Crouzel, La CFDT fait disparaitre près de 30\% de ses adhérents avant son congrès, Le Figaro, 15 mai 2018.

${ }^{12}$ Durant cette période une vingtaine d'enquêtes ont été réalisées par D. Andolfatto, J.-C. Basson, A. Bevort, M. Casula, T. Choffat, M. Croisat, J. Derville, G. Henry, D. Labbé, P. Mathiot, G. Nezosi, L. Olivier, J. Olmi, F. Paccou, P. Pons, G. Prince, A. Rey, J.-Y. Sabot, B. Verrier. Certains de ces rapports peuvent être trouvés en ligne, notamment deux rapports de synthèse ; Dominique Labbé. Syndicats et syndiqués en France depuis 1945, Paris, L'Harmattan, 1996; Dominique Andolfatto et Dominique Labbé. Les syndiqués en France. RueilMalmaison, Liaisons sociales, 2007.

${ }^{13}$ DARES. art. cité, 2016, p 2.

${ }^{14}$ La principale justification de ce coup de hache serait que, dans les adhérents (numérateur du taux de syndicalisation), il y aurait des retraités alors que le dénominateur ne comporte que la population active salariée. Il faudrait donc retirer ces retraités du numérateur. Selon la note de la DARES de 2004, ces retraités représentent $20 \%$ des effectifs, en proportion stable de la Libération aux années 1990. Dans la note de 2016, cette proportion a été ramenée à 14\% (également stable de 1949 et la fin des années 1990). Ces affirmations traduisent une méconnaissance du syndicalisme. Premièrement, les retraités n'ont jamais pesé aussi lourd (sauf dans quelques fédérations comme les cheminots, l'énergie ou les charbonnages) et, jusqu'aux années 1970, ce poids était négligeable. De plus, l'estimation du nombre d'adhérents est faite à l'aide des timbres encaissés par les trésoreries confédérales, or les retraités ne paient qu'un timbre par trimestre contre un par mois pour les actifs. Autrement dit, en admettant ces taux excessifs de $20 \%$ ou (14\%), c'était un tiers (soit 6,7\% ou 4,7\%) qu'il aurait fallu défalquer.
} 
impatience une relance des luttes et par ceux qui ont imaginé la "rénovation de la démocratie sociale française".

La série originale ${ }^{15}$, mutilée par le ministère du Travail, contredit ce story-telling. Elle commence en 1949 (faute de données fiables sur la population active à la Libération) mais il est admis que, entre 1945 et 1947, un salarié sur deux est syndiqué et que la baisse s'amorce en 1948. Elle conduit à deux conclusions principales que les statisticiens du ministère du Travail ont souhaité effacer.

Premièrement, entre les années 1950 et 1978, la syndicalisation oscille entre 24 et $30 \%$. Ajoutons que la fidélité des adhérents a été assez grande (l'adhésion durait en moyenne une dizaine d'années). Certes ce taux était inférieur à celui de l'Angleterre, de l'Allemagne ou des pays nordiques mais c'était plus qu'aux Etats-Unis ou en Italie. Il est donc faux d'affirmer que la syndicalisation a toujours été faible en France. De plus, durant ces trente années, la population active salariée a augmenté rapidement (de 10 à 17 millions de salariés), elle s'est rajeunie, s'est féminisée et son niveau d'éducation a nettement augmenté. Enfin, l'emploi tertiaire est devenu prépondérant. Le simple maintien du taux de syndicalisation signifie que les syndicats de cette époque ont été capables de gagner des adhérents et de s'adapter aux changements de l'économie et de la société française. De fait, les enquêtes citées ci-dessus qui ont été réalisées dans des entreprises, des unions départementales et des fédérations - ont montré que, durant ces trente années, il a existé un syndicalisme vivant, centré sur les lieux du travail et essentiellement consacré à apporter des aides aux salariés dans leurs difficultés quotidiennes au travail tout en donnant une voix aux exclus du système.

Des années 1980 à aujourd'hui, la syndicalisation a été divisée par 4 (de 30\% à 7\%). Il s'est donc produit un profond divorce entre les syndicats et les salariés français. Les principales raisons de ce divorce sont la disparition de la plupart des syndicalistes des lieux du travail et leur incapacité à apporter une aide individuelle efficace aux salariés comme le faisaient leurs aînés. C'est aussi la conséquence de l'institutionnalisation et de la professionnalisation du syndicalisme : les syndicalistes sont de plus en plus absorbés par des tâches bureaucratiques et de représentation et sont moins présents dans les collectifs de travail.

Deux institutions ont particulièrement souffert de ces changements : les délégués du personnel - qui jouaient autrefois le rôle de défenseur du salarié face à la hiérarchie - et les délégués au comité d'hygiène et de sécurité (CHS-CT), héritiers de la plus ancienne

\footnotetext{
${ }^{15}$ Ces données sont présentées dans : Anfolfatto Dominique, Labbé Dominique. Le Débat, 142, novembre-
} décembre 2006, p. 119-132. 
institution de représentation du personnel (les délégués mineurs). Naturellement, ces deux fonctions n'avaient d'utilité que si leur titulaire travaillait au milieu des salariés qu'il représentait et que sa valeur professionnelle ne pouvait être mise en doute. C'est pourquoi ces deux fonctions ont particulièrement souffert du cumul des mandats et de la professionnalisation du syndicalisme. De fait, la fusion de toutes les institutions de représentation du personnel dans une instance unique - le comité social d'entreprise institué par les ordonnances de 2017 - entraînera un nouvel affaiblissement de ces deux fonctions qui sont pourtant les plus utiles aux salariés, mais que le patronat juge gênantes et coûteuses, du moins quand elles ne fonctionnent pas de manière purement formelle. Le CSE répond aussi à d'autres objectifs, notamment "rationnaliser" le dialogue social et impliquer davantage les élus du personnel dans la "gouvernance" de l'entreprise.

Déjà la négociation d'entreprise s'est considérablement développée et aboutit à un grand nombre d'accords collectifs dont les principaux objectifs sont la diminution des charges salariales, l'augmentation de la flexibilité des salariés et du taux d'utilisation des équipements ${ }^{16}$. Pour les employeurs, comme pour les responsables politiques, cette déréglementation présente un avantage : les salariés sont supposés y consentir par la voix de "leurs" syndicats. Cela explique aussi le paradoxe suivant ${ }^{17}:$ des syndicats de plus en plus présents dans les entreprises - en tant qu'institution - mais de moins en moins sollicités par les salariés qui s'en méfient.

Plus au fond, la France a changé de modèle syndical. Alors que les syndicats perdaient leurs racines sociales, leurs appareils connaissaient une croissance considérable grâce à des personnels mis à disposition et à de nouvelles sources de financement.

\footnotetext{
${ }^{16}$ Voir le bilan annuel de la négociation collective publié par le ministère du Travail. Ce bilan, très factuel, ne s'intéresse guère au contenu des accords. Voir également : Josépha Dirringer, L'esprit du dialogue social : de la loi du 20 août 2008 aux accords collectifs relatifs au droit syndical et à la représentation des salariés, Revue de l'IRES, 87, 2015-4, p. 125-151. Dominique Labbé et Gilles Nezosi. Négociation collective et paritarisme : regards sur les relations professionnelles et la protection sociale. In Dominique Andolfatto (dir.). Les syndicats en France. Paris, la Documentation française, 2013, p. 149-186.

17 Olivier Jacod. Les institutions représentatives du personnel : davantage présentes, toujours actives mais peu sollicitées par les salariés, DARES, Premières informations et premières synthèses, $\mathrm{n}^{\circ}$ 05-1, février 2007.
} 


\section{Gonflement de l'appareil}

En 1974, alors que la CFDT comptait 750000 adhérents, tous de chair et d'os (soit près de 5\% des salariés de l'époque), une quarantaine de personnes travaillaient à la confédération, tous salariés de l'organisation. Aujourd'hui, 260 personnes travaillent à la seule confédération (boulevard de Belleville) ${ }^{18}$ alors que la CFDT revendique 630000 adhérents (et n'en compte en réalité que moins d'un demi-million soit $2 \%$ des salariés). Autrement dit, alors que le nombre des adhérents diminuait d'au moins un tiers, l'appareil confédéral a été multiplié par 6,5. Lorsqu'on peut obtenir des données sur les unions régionales ou les fédérations, on constate le même gonflement des appareils.

D'après les comptes publiés par la CFDT, la masse salariale correspond à une centaine de personnes. Les autres sont donc "mis à disposition" de la confédération par des entreprises, des administrations publiques ou d'autres entités extérieures au périmètre comptable de la confédération. Les informations disponibles montrent, à tous les niveaux, la prépondérance des "mis à disposition" qui continuent à être payés par leurs administrations ou entreprises tout en étant permanents syndicaux.

Au cours de la même période l'appareil de la CGT a quintuplé alors que ses effectifs passaient de 1,8 millions d'adhérents à 550 000. Là encore, la source principale de ce gonflement provient des mises à disposition. Roger Lenglet et Jean-Luc Touly ${ }^{19}$ citent à ce propos le cas de la fédération CGT de la construction, secteur d'activité qui pèse lourd dans l'économie française (1,8 millions d'emplois en 2014). La fédération avait 120000 syndiqués en 1974 (tous en chair et en os) et 10000 en 2012. Dans le même temps, le nombre des permanents travaillant à la fédération est passé de 4 à 30. Autrement dit, l'appareil central a été multiplié par sept alors que le nombre de syndiqués était divisé par douze. Dix de ces permanents étaient mis à disposition par Delkia (filiale de Veolia).

L'enquête de Lenglet et Touly ne s'arrête pas à ce constat numérique, elle révèle le prix à payer pour cette "générosité" patronale. Dans les années 1990, plusieurs syndicalistes CGT, qui avaient dénoncé des pratiques douteuses dans l'entreprise, ont été privés de leurs mandats syndicaux, licenciés par l'entreprise, traînés en justice par la CGT pour avoir voulu créer un autre syndicat ${ }^{20}$.

\footnotetext{
18 Jacques Chérèque. Si on me cherche. Paris, Albin Michel, 2008. L'auteur était secrétaire général de la confédération.

${ }^{19}$ Roger Lenglet, Jean-Luc Touly. Syndicats : corruption, dérives, trahisons. Paris : First, 2013, p 180-181.

${ }^{20}$ Roger Lenglet et Jean-Luc Touly, op. cit., p. 169-180.
} 
Quel est le nombre de ces personnels "mis à disposition" ? Dans le secteur public, quelques rapports - notamment de la Cour des comptes, sur certaines administrations comme La Poste, la Banque de France, la police, l'éducation nationale, et quelques entreprises nationales comme EDF ou la SNCF ainsi qu'une enquête sur deux départements de province ${ }^{21}$ ou certains débats parlementaires comme à l'occasion de la dernière réforme ferroviaire aboutissent à la conclusion que les syndicats disposent au minimum de 30000 à 40000 équivalents temps, sans qu'il soit possible de chiffrer le nombre de décharges complètes. Dans le secteur privé, il est impossible de connaître le nombre de ces décharges - probablement équivalent à celui du secteur public - le législateur ayant omis de prévoir une obligation de déclaration en même temps qu'il légalisait cette pratique potentiellement assimilable à un abus de bien social. Sur ce point, il y a une certitude : tous les accords collectifs passés dans les grandes entreprises françaises prévoient ces mises à disposition de personnels pour leurs syndicats représentatifs ainsi que l'allocation de moyens financiers et matériels importants (locaux, communication, reprographie, défraiements des déplacements). Comme dans la fonction publique, ces accords comportent également le versement d'une subvention annuelle en fonction du nombre de voix obtenues aux élections professionnelles ${ }^{22}$.

Si les mises à disposition de personnel constituent la principale ressource, elles sont impossibles à chiffrer précisément, le législateur n'ayant pas exigé qu'elles apparaissent dans les comptes des syndicats alors même qu'il légalisait cette pratique et qu'il votait le principe de la "transparence" de ces comptes. En évaluant cette ressource aux salaires versés à ces permanents, la commission Perruchot était parvenue à la conclusion que les cotisations ne représentaient plus que 4 à $5 \%$ du total de l'économie du syndicalisme ${ }^{23}$.

En dehors de ces moyens cachés, la première ressource des cinq confédérations provient d'un impôt sur les salaires créé - à bas bruit - par la loi de mars 2014, relative à la formation professionnelle, à l'emploi et à la démocratie sociale. En 2017, cet impôt a rapporté 93,2

\footnotetext{
${ }^{21}$ Desforges Corinne et al. Rapport sur le bilan des moyens alloués aux organisations syndicales dans la fonction publique. Analyse détaillée dans deux départements (le Rhône et le Loiret). Ministère de l'intérieur et Inspection générale des services. Juin 2010 (consultable en ligne sur le site du ministère de l'Intérieur). Le rapport recommandait une simplification, une uniformisation et la publication de ces moyens. Il est resté lettre morte.

${ }^{22}$ A titre d'exemples, voir les accords analysés par Josépha Dirringer (art. cité).

${ }^{23}$ Nicolas Perruchot. Rapport sur les mécanismes de financement des organisations syndicales d'employeurs et de salariés. Assemblée nationale, novembre 2011. Pour la première fois depuis 1875, un rapport d'une commission d'enquête parlementaire n'a pas été publié (cependant, il est consultable en ligne : http://www.lepoint.fr/html/media/pdf/rapport-perruchot.pdf).
} 
millions d'euros auxquels se sont ajoutés 32,6 millions de subventions versées par l'Etat ${ }^{24}$ (en comparaison les partis politiques ont reçu une aide publique de 63 millions d'euros en 2017). Ces sommes sont partagées entre les organisations représentatives des employeurs (Medef, CGPME, UPA) qui ont reçu 40 millions d'euros, et les organisations syndicales représentatives des salariés qui se sont partagé 83,3 millions d'euros ${ }^{25}$.

Curieusement, personne n'a semblé remarquer que cet impôt équivaut à une cotisation obligatoire versée par les salariés aux syndicats et que, de plus, il oblige ces salariés - même les plus pauvres - à financer les organisations d'employeurs qui a priori ne sont pas conçues pour défendre leurs intérêts. De plus, ces organisations - syndicales ou patronales - disposent de ces ressources fiscales sans avoir de comptes à rendre sur leur utilisation ${ }^{26}$.

Viennent ensuite les cotisations des adhérents qui, dans aucune des cinq confédérations représentatives, ne dépassent le tiers des recettes figurant au bilan. Encore faut-il préciser que beaucoup de timbres encaissés par les trésoreries confédérales ne proviennent pas d'adhérents en chair et en os mais de financements patronaux (le plus connu est le "chèque AXA" créé par une convention de 1990 avec la CFDT, la CFTC et la CGC) ${ }^{27}$ ou de prélèvements sur les organismes gérés par les syndicats (en premier lieu les comités d'entreprise). Une affaire récente est venue jeter un peu de lumière sur ces pratiques. Une cotisation obligatoire de $0,2 \%$ du salaire, prélevée sur les salariés agricoles, est partagée entre la FNSEA et les syndicats représentatifs. La Fédération CGT agro-alimentaire et forestière perçoit à ce titre 1,1 millions d'euros dont la moitié seulement apparaît dans ses comptes publiés. Selon les déclarations du président de cette fédération, une partie de l'autre moitié aurait servi à payer "des cotisations

\footnotetext{
${ }^{24}$ Association de Gestion du Fonds Paritaire National. Rapport annuel 2017 sur l'utilisation des crédits du Fonds pour le financement du dialogue social. Paris, $1^{\mathrm{er}}$ octobre 2018. Le rapport de l'AGFPN est consultable en ligne sur son site.

${ }^{25}$ CGT : 18,9 millions ; CFDT : 18,6 ; FO : 15,0 ; CGC ; 12,6 ; CFTC : 12,5 ; UNSA : 3,0 et SUD : 2,6 millions.

${ }^{26}$ Le rapport de l'AGFPN se contente d'indiquer les sommes versées sans aucune mention de l'utilisation de ces sommes. Cette absence de justification est soulignée dans un rapport de la Cinquième chambre de la Cour des comptes (La mise en auvre de la réforme du financement du paritarisme, 26 décembre 2017). Bien que ce rapport fasse preuve d'une extrême modération dans les termes employés et dans ses recommandations, le président de chambre n'a pas jugé utile de le publier et a mis fin au contrôle de la Cour.

${ }^{27}$ Rémy Bourguignon, Mathieu Floquet, When union strategy meets business strategy: The union voucher at AXA, Business History, 2019, vol. 61, n 2, p. 260-280.
} 
gratuites à quelques milliers d'adhérents" 28 .

Pour les différentes confédérations, ces cotisations sans réels cotisants représentent au moins un timbre sur cinq encaissés, voire un timbre sur quatre, de telle sorte que les adhérents en chair et en os ne dépassent pas les 500000 à la CGT ou à la CFDT et les 300000 à FO, dont une bonne partie sont des permanents, des élus ou des mandatés. Les adhérents "virtuels" présentent beaucoup d'avantages sur les vrais : ils ne posent pas de questions, votent toujours comme il faut et ne contestent jamais les dirigeants, ni la ligne de l'organisation. Ils permettent également d'afficher une quasi-stabilité des effectifs. Enfin, les cotisations étant la mesure de l'indépendance d'une organisation, il est facile de comprendre pourquoi beaucoup de directions syndicales consentent quelques sacrifices financiers pour "recycler" en cotisations certaines ressources et embellir ainsi leur bilan.

\section{Conclusion}

En France, l'indépendance syndicale ressemble à une sorte de "village Potemkine". Une grande partie des salariés ne sont sans doute pas dupes de cette situation, même s'ils ne disposent que de peu d'informations à ce sujet. Dès lors, comment ne pas y voir une des origines de la crise actuelle?

La situation du syndicalisme français illustre plusieurs des difficultés hexagonales. Parmi celles-ci - outre le discrédit des représentants aux yeux des représentés et le gaspillage des ressources publiques - l'échec récurrent de réformes engagées sans connaissance de la réalité que l'on prétend réformer ou pire, fondées sur des données fausses fabriquées pour coller à l'idéologie du moment.

Si ces tentatives n'ont pas atteint les objectifs proclamés, elles ne sont pas pour autant sans portée. Ainsi à la faveur de multiples réformes sociales lancées au cours des dix dernières années, le cartel des "partenaires sociaux" est parvenu à interdire de facto l'émergence de nouveaux syndicats, à obtenir la légalisation de ressources assimilables à des abus de biens sociaux (ou de prise illégale d'intérêts), comme les mises à disposition de

\footnotetext{
${ }^{28}$ Alain Guédé. Quand les saisonniers récoltent de l'oseille pour la CGT, Le Canard enchaîné, 11 juillet 2018. La famille Huck dirige la FNAF depuis plus de 40 ans. C'est la seule fédération CGT à être restée membre de la FSM (Fédération Syndicale Mondiale de l'ex-bloc communiste). D'après l'enquête déjà citée, réalisée pour le ministère du Travail en 2006, la FNAF avait réellement 21000 adhérents au début des années 2000. Le demimillion d'euros de cotisations représente près de 5000 adhérents fictifs - soit un cinquième des effectifs - et un assez faible encaissement (en théorie la fédération perçoit un peu moins de $30 \%$ des cotisations, le reste étant versé aux syndicats, aux unions interprofessionnelles et à la confédération). Il faut donc chercher des motifs autres que financiers à ce genre d'opérations.
} 
personnel ou les aides financières. Puis ce cartel a fait instituer en sa faveur un nouvel impôt sur les salaires sans avoir à rendre compte de l'utilisation de ces fonds. Enfin, il a consolidé son emprise sur de multiples organismes paritaires sur lesquels il opère des prélèvements tout aussi peu transparents.

Dans les entreprises, les syndicalistes tiennent également un rôle incontournable pour l'adaptation des normes du travail. Jamais, ils n'ont été aussi souvent consultés par les pouvoirs publics et associés à tous les niveaux à la décision. Leurs leaders sont devenus des figures de la vie politique et, tels ceux des partis politiques classiques, ils se prennent au jeu de celle-ci ${ }^{29}$. Ces évolutions ont conduit les syndicats à renoncer aux fonctions qu'ils tenaient traditionnellement dans le monde du travail et la société. Certes, leurs rôles juridiques ont grandi et se sont technicisés ; leurs cadres sont devenus, selon une expression de Pierre Rosavallon, des "fonctionnaires du social". Mais les liens sociaux qu'ils cultivaient, la fonction tribunitienne qu'ils assumaient, la société qu'ils soutenaient, se sont dissipés quand ils ne se sont pas évanouis. Dans une large mesure, la "marée jaune" de cet hiver français du mécontentement est une conséquence de ce changement de nature du syndicalisme, de sa professionnalisation et de sa clôture. Ce mouvement social de l'hiver 2018 restera d'autant plus dans l'histoire qu'il aura été le premier à être sinon antisyndical, du moins a-syndical. Le rejet est même apparu réciproque. Manifestement, une page s'est tournée.

\footnotetext{
${ }^{29}$ Voir par exemple le long article de François Fressoz consacré à Laurent Berger, secrétaire général de la CFDT : "Comment Laurent Berger est devenu le premier opposant à Emmanuel Macron", Le Monde, 9 mars 2019.
} 